\title{
Primary Immunodeficiencies Diseases (Pid) In Senegalese Children: A Cross Sectional Observational Study
}

\section{Awa Kane ( $\nabla$ cawa.kane@gmail.com )}

Albert Royer children's hospital https://orcid.org/0000-0003-4796-7211

Indou Deme-Ly

Cheikh Anta Diop University of Dakar, Albert Royer children's hospital

\section{Mame Sokhna Gueye}

National Blood Transfusion Center

\section{Babacar Niang}

Cheikh Anta Diop University of Dakar, Albert Royer children's hospital

\section{Aminata Mbaye-Dieyla}

Albert Royer children's hospital

\section{Guilaye Diagne}

Albert Royer Children's hospital

\section{Aliou Mar Coundoul}

Albert Royer Children's hospital

\section{Yaye Joor Dieng}

Cheikh Anta Diop University of Dakar, Albert Royer children's hospital

\section{Diénaba Fafa Cissé}

Cheikh Anta Diop University of Dakar, University hospital center of Pikine Idrissa Demba Ba

Cheikh Anta Diop University of Dakar, Albert Royer children's hospital

\section{Aliou Thiongane}

Cheikh Anta Diop University of Dakar, Albert Royer children's hospital

\section{Abou Ba}

Cheikh Anta Diop University of Dakar, Albert Royer children's hospital

\section{Aminata Nakoulima}

Principal hospital of Dakar

\section{Aminata Diouf}

University hospital center of Pikine

\section{Younoussa Keita}

Cheikh Anta Diop University of Dakar, Aristide Le Dantec University hospital center

\section{Pape Moctar Faye}


Cheikh Anta Diop University of Dakar, Albert Royer children's hospital

\section{Amadou Lamine Fall}

Cheikh Anta Diop University of Dakar, Albert Royer children's hospital

\section{Assane Sylla}

Cheih Anta Diop University of Dakar, Aristide Le Dantec University hospital center

\section{Ibrahima Diagne}

University of Gaston Berger, Saint Louis Regional Hospital

\section{Ousmane Ndiaye}

Cheikh Anta Diop University of Dakar, Albert Royer children's hospital

\section{Tandakha Ndiaye Dièye}

Cheikh Anta Diop University of Dakar, National Blood Transfusion Center

\section{Research note}

Keywords: Primary immunodeficiencies diseases (PID), children, sub-Saharan Africa

Posted Date: October 20th, 2020

DOI: https://doi.org/10.21203/rs.3.rs-71419/v1

License: (1) (1) This work is licensed under a Creative Commons Attribution 4.0 International License. Read Full License 


\section{Abstract \\ Objective:}

Primary immunodeficiencies are rarely related in sub-Saharan Africa. The aim of this study was to identify their different clinical patterns, to describe their biological phenotypes and to analyse treatment. A cross-sectional observational study over a period of 4 years and 2 months in 5 Senegalese paediatric departments was conducted. Children were recruited according to the recommendations of African society for immunodeficiencies (ASID) or the 10 warning signs for PID's diagnosis. Biological explorations were performed in these patients.

\section{Results:}

30 cases of PID were recorded. The sex ratio was 1:1; with a median age at diagnosis of 24 months. The most common clinical patterns were respiratory infections (18 patients) including tuberculosis pneumonia in almost one-third of cases (22\%), followed by digestive and then cutaneous manifestations. This signs occurs in a specific context; as inbreeding (36,7\% of cases), death in sibling (30\% of cases), or previous hospitalizations $(66,7 \%)$. Biological exploration made it possible to obtain immunological confirmation in 10 children, including one genetically confirmed. The most common category of PID was syndromic PID. Treatment was based mainly on antibiotic prophylaxis. PID are a reality in Senegal, but are certainly underestimated. Continue recruitment will enable advocacy for access to certain treatment.

\section{Introduction}

Primary immunodeficiencies diseases have often been considered rare, and several diagnostic tools and approaches have been developed to facilitate diagnosis $[1,2,3,4,5]$. However, their prevalence is underestimated. [6, 7] Primary immunodeficiencies diseases occurs most often in children [8].

In Africa, data on recorded PID came mainly from the Maghreb and South Africa. In 2014, the establishment of the first report of the Moroccan register covering a period of 15 years identified 421 PID cases in children and adults [8]. In the same year, in South Africa, the incidence of PID in paediatrics was estimated between 5 and 15 new cases per year [9]. The place occupied by these PID in childhood diseases in Senegal is not yet elucidated.

The aim of this study was to prove the existence of PID in children. The specific objectives were to identify the different clinical patterns presented by these patients, to describe the different immunological phenotypes and to propose management.

\section{Methods}

Study design 
It was a multicenter observational cross-sectional study over a period of 4 years, from October 1, 2014 to December 1, 2018, initiated by the Immunology Laboratory of the National Blood Transfusion Center (CNTS) in partnership with 5 paediatric services. The 4-step approach of B. Admou and al. has been adopted [3].

Patients:

We included children aged 0 to 15 years, who had the following criteria:

Lack of evidence for secondary immunodeficiency with, in particular, negative HIV test in the child or mother (children $<18$ months) and:

- The presence of at least two of the 10 warning signs of PID, or

- The existence of signs described in the 10 recommendations for PID diagnosis proposed by the Moroccan and the African Society for Immunodeficiencies (ASID), 2015.

We excluded: children who had malnutrition, proven chronic disease (e.g.: sickle cell disease, systemic diseases), long term corticotherapy or other immunosuppressive therapy and children who did not have parental advisory.

Variable:

A data collection form was established for each included patient. It included general data, clinical data, biological data, classification of PID using the IUIS classification 2017, determination of the probability score by ESID criteria into 3 categories (definitive, probable or possible), treatment and evolution.

\section{Statistical analysis:}

The data collected were recorded and analysed using the SPSS software version 24 . A $P$ value less than 0.05 was deemed statistically significant.

\section{Results}

A total of 30 cases of PID were recorded with an equal distribution between girls and boys. Patients were less than 5 years old in $80 \%$ of cases (26 cases) with a median age of 24 months, extremes were 1 month and 156 months.

Hospitalization services, recorded $70 \%$ of cases or 21 patients. The specialized consultation was represented at $13 \%$, exclusively by the paediatric dermatological consultation.

Inbreeding was found in more than a third of patients (11 cases or $36,7 \%$ ) and table 1 presents different personal and family histories of suspected cases of PID.

Table 1: Family and personal history of PID cases 


\begin{tabular}{|lll|}
\hline Anamnestic data & Number & Frequency (\%) \\
\hline Death in the sibling & 9 & 30 \\
Similar cases & 2 & 6,7 \\
Previous hospitalizations & 20 & 66,7 \\
Recurrent ENT infections & 4 & 13,3 \\
\hline
\end{tabular}

\section{Clinical patterns during PID}

Respiratory and/or ENT manifestations

Representing the most common manifestations, they were exclusively related to infectious process (22 patients or $73 \%$ ). Among them four patients had pulmonary tuberculosis. Pneumonia was associated with other infection sites, in 9 patients with a combined PID.

3 cases of humoral immunodeficiency were suspected in patients with recurrent pneumonia. We noted staphylococcal pleuropneumonia associated with a neutrophilia of $52000 / \mathrm{mm} 3$ as part of an innate PID; in a 2 month old infant.

Digestive manifestations

They concerned a third of patients, with recurrent acute gastroenteritis (4 cases) and chronic gastroenteritis ( 1 case). The digestive haemorrhages found in 2 patients suggested a predominantly antibody deficiency. This was a 13-year-old boy with inflammatory Bowel disease on colonoscopy and a 2 years old boy, who had identical manifestations, but did not perform colonoscopy because of severe growth retardation. 3 patients had persistent thrush, one of them is highly suspect of chronic mucocutaneous candidiasis (CMC).

\section{Skins disorders}

It was the third manifestations and concerned 8 patients (26\%). Among these patients, 4 were referred to us by the paediatric dermatological consultation: 2 cases of hereditary angioedema, one case of Wisckott Aldrich syndrome (WAS) (Image 1 and 2); and 1 case of MHC class II deficiency with repeated skin abscesses. A patient with ataxia-telangiectasia syndrome presented ocular telangiectasias (Image S1). Skin manifestations led to a definitive or probable diagnosis in 3 out of 4 cases.

Neurological manifestations

Seven patients had neurological manifestations. Five patients presented bacterial meningitis. We also observed a familial case of ataxia-telangiectasia syndrome in 2 sisters aged 9 and 3 years with 2 cases of death in the siblings and parental inbreeding. In addition to ataxia and ocular telangiectasia, both had presented several infections (annex/Table S3). Biological analysis confirmed the diagnosis with a 
combined deficiency at lymphocyte immunophenotyping. Their 24-month-old younger brother was included because of recurrent pneumonia. He did not have ataxia or telangiectasia, however, a combined immune deficiency status has been established.

Failure to thrive:

It was the main impact of the clinical manifestations of PID as observed in table S1.

Others manifestations:

We found microcephaly and a " bird like face » in a patient, compatible with Bloom syndrome. 3 patients presented, sepsis during the evolution of their symptoms.

\section{Biologics finding}

Blood accounts were performed in all patients. Two thirds of patients (20 patients) had lymphocyte immunophenotyping and $56.7 \%$ or 17 patients had serum protein electrophoresis. The weight determination of immunoglobulins and the determination of serum complements were performed in 4 patients. The genetic study was performed in one patient.

First-line biological exploration:

Contribution of blood account to the diagnosis of PID in our study: in our patients lymphopenia was present in $21 \%$ of cases ( 6 patients) and was systematically related to a combined deficiency.

Neutropenia was also found in 6 patients, including 1 case of congenital neutropenia. Two patients had persistent and major hyperleukocytosis compatible with phagocytosis function abnormalities. Low platelets with small size were found in the patient with WAS. (Annex/ Table S3)

Serum protein electrophoresis was normal (50\% of cases) or showed hypergammaglobulinemia ( $42.8 \%$ of cases); only one patient developed hypogammaglobulinemia as part of a combined immune deficiency.

The weight determination of immunoglobulins and the determination of serum complements $(\mathrm{C} 3, \mathrm{C} 4$, and $\mathrm{CH} 50$ ) performed outside the country were normal.

Specific immune exploration:

At lymphocyte immunophenotyping, CD4 T cells were decreased in one-third of cases (10 patients). The decrease in CD8 $\mathrm{T}$ lymphocytes was found in $17.4 \%$ of cases. CD19 B lymphocyte reduction was present in $30 \%$ of cases, or 6 patients. This reduction in B cells was not isolated and was always associated with a reduction in $T$ cells as part of a combined deficiency.

The dosage of $\mathrm{C} 1$ inhibitor serum complements performed as part of a hereditary angioedema has returned to normal. 
Genetic study:

Molecular biology was performed in only one patient and confirm the mutation of the WAS gene.

\section{Aetiology of primary immunodeficiencies}

We identified 6 categories of PID (Table S2) according to clinical pattern, biological finding, and/or genetic study. The most common PID category was well-defined syndromes (20\%).

The undetermined PID (8 patients) were patients who responded to different categories of PID according to clinical pattern but had normal or incomplete biological exploration to classify them.

Our study included 30 cases of PID (Annex/ Table S3). Among these patients, 10 had specific immunological confirmation including one with genetic confirmation, it was a WAS. Twenty patients are considered as possible diagnosis of PID because their clinical and biological signs were insufficient. Of these patients, half had normal immunological exploration and the other half had incomplete exploration.

Based on the PID identified in our study, the suspected genetic transmission modes were dominated by autosomal recessive transmission (65\%).

\section{Treatment}

Thirteen patients (46.6\% of cases) were started on Sulfamethoxazole/Trimethoprim antibiotic prophylaxis. Therapeutic abstention was observed in equal proportions, particularly in patients with a possible diagnosis (ESID criteria).

In the management of bacterial infectious complications, antibiotic therapy was systematic including antituberculosis drugs. Intravenous infusion of immunoglobulins (IVIG) was offered to our patients, however this treatment was not effective due to lack of resources.

Hematopoietic stem cell transplantation was not available during our study.

\section{Evolution}

We recorded 5 cases of death, including 2 in the same family. All the deceased patients had a combined immunodeficiencies.

We did not find a statistically significant association between definitive and probable diagnosis of PID and some qualitative variables such as inbreeding, previous hospitalizations or age.

\section{Discussion}

In our study, 30 children were enrolled over a 4-year period, with an average of 7.5 cases/year. However, our study did not cover all university hospitals in Senegal. In Morocco, Bousfiha and al. recordered 401 cases of PID with a mixed sample including adults and children [8]. In South Africa, the incidence of PID 
in paediatrics was estimated between 5 and 15 new cases per year, which was superimposed on our study [9].

Our median age at diagnosis was 24 months, similar to Morocco (24 month) and Sweden (28 month) [8, 10]. In France, the median age was 3.3 years (39 months), and included both children and adults [11]. We did not assess the diagnostic delay because it was poorly defined by the parents. However, $60 \%$ of cases, had one to several previous hospitalizations, which could indirectly indicate the diagnostic delay.

Several clinical manifestations have been described, the main one, being respiratory and/or ENT infections (22 patients) followed by digestive manifestations, then staturo-ponderal growth retardation and dermatological manifestations. These results were superimposed on those found in Europe $[12,13]$.

Blood count cell is a simple and inexpensive test that provided useful information for referral to a given PID. Indeed lymphopenia was specific to combined PID in our study. Neutrophilia, found was compatible with phagocytosis abnormalities such as chronic granulomatosis disease and Leukocyte Adhesion Deficiency (LAD). It would have been beneficial to have functional tests for phagocytosis to confirm the suspected types of PID.

Brodsky and al. show, PID in children with less than a half (7 patients) who had genetic and/or molecular confirmation. Therefore, although genetic confirmation is the definitive diagnosis (probability score > $98 \%$ ), specific immune exploration should be accessible because it allows the diagnosis (probability score $>85 \%$ ) and legitimate starting treatment. In our study, immunophenotyping led to the diagnosis in 10 patients and only one of them had the genetic confirmation.

Well-defined syndrom was the most common category of PID of our study, as found in the Maghreb [8, $14,15]$; while in developed countries the predominantly antibodies deficiency was the most common category $[12,16,17]$. This contrast could be explained by the fact that well-defined syndromes are characterized by specific clinical sign that attract the attention of doctors. Second, the predominantly antibodies deficiency are mainly linked to the $\mathrm{X}$ chromosome, and therefore not influenced by inbreeding which is more found in the Middle East and Africa $[8,18,19,20]$.

Almost a half of patients received antibiotic prophylaxis, in others series IVIG were the primary treatment with or without bone marrow transplantation $[12,16,21,22]$. This lack, is mainly due to the limited accessibility of intravenous immunoglobulins that are not subsidized.

\section{Conclusions}

Primary immunodeficiencies in children are widely underestimated in Senegalese hospitals. PID diagnosis is accessible considering specific clinical pattern, particular family history, abnormalities in blood count cells particularly: lymphopenia, neutropenia, neutrophilia. The access to specific immunological explorations will facilitate diagnosis for paediatricians. Continue recruitment of new 
patients will allow us to refine our epidemiological profile and advocate for the access to certain treatments such as IVIG or bone marrow transplants.

\section{Limitation}

The limits were related to the sample size of 30 cases, which was a constraint in the analysis of the associated factors. The second limitation was biological exploration, which was not uniform in our patients. Indeed, not all patients had a complete immunological assessment due to the lack of reagents.

\section{Abbreviations}

CGD

Chronic Granulomatous Diseases

$\mathrm{CMC}$

Chronic Mucocutaneous Candidiasis

IL12

Interleukin 12

INFY

Interferon gamma

IVIG

Intravenous Immunoglobulins

LAD

Leucocytes Adhesion Deficiency

NJS

Nijmegen syndrome

PID

Primary immunodeficiencies Disease

SCID

Severe Combined Immunodeficiency

WAS

Wiskott Aldrich syndrome

\section{Declarations}

\section{Ethics approval and consent to participate}

This study was approved by the ethical committee of Cheikh Anta Diop University of Dakar.

Written consent was obtain from all the participants of the study.

This consent was obtain from a parent of any participants under the age of 16 . 


\section{Consent for Publication}

Written informed consent was obtain from the parents of the patients for publication of the study and any accompanying images.

\section{Availability of data and materials}

The datasets used and analysed during this study are available from the corresponding author on reasonable request.

\section{Competing interests}

The authors declare no conflict of interest.

\section{Funding}

The authors declare that they have not received another source of funding

\section{Authors' contributions}

D. and O.N. have conceptualized the project. All the others co-authors participated in the data collection from patients at the hospital where they are affiliated, with the support of A.K., I.D.L and M.S.G. AK and MSG analysed data. AK drafted the manuscript. All authors reviewed, edited, and approved the final manuscript.

\section{Acknowledgements}

Not applicable.

\section{References}

1. M. Boyle, R. H. Buckley. Population Prevalence of Diagnosed Primary Immunodeficiency Diseases in the United States. J clin Immunol 2007 ; 27 (5) : 497-502. DOI: 10.1007/s10875-007-9103-1.

2. http://www.worldpiweek.org/sites/default/files/article_docs/Editorial\%202016\%20WPIW_final.pdf.

3. Admou B, Haouach K, Ailal F, Benhsaine I, Barbouche MR, Bejaoui M, A. A Bousfiha. Deficits immunitaires primitives : approche diagnostique pour les pays émergents. Immuno-analyse et biologie spécialisée 2010 ; 25 : 257-265. https://doi.org/10.1016/j.immbio.2010.09.003

4. http://www.asid-africa.org/ images/pdfs/recommendations_french.pdf

5. http://www.esid.org/workingParties/clinical/Ressources/Diagnostic-criteria-for-PID2

6. Kobrynski L, Powell RW, Bowen S. Prevalence and morbidity of primary immunodeficiency Diseases, United States 2001-2007 J Clin Immunol. 2014; 34 (8): 954- DOI: 10.1007/s10875-014-0102-8 
7. Avni Y. J, Vivek N. I. , John B. H, Jennifer L.S.S, et coll. Incidence and Temporal Trends of Primary Immunodeficiency: A Population-Based Cohort Study. Mayo Clin Proc. 2009 Jan; 84(1): 16-22.

8. Bousfiha AA, Jeddane L, El Hafidi N, Benajiba N, Rada N, El Bakkouri J. First report on the Moroccan registry of primary immunodeficiencies: 15 years of experience (1998-2012). J Clin Immunol. 2014; 34(4):459-68. DOI:1007/s10875-014-0005-8

9. Eley B \& Esser M. Investigation and management of primary immunodeficiency in South African children S. Afr Med J. 2014; 104 (11): 73-04. DOI:7196/samj.8946

10. Brodszki N, JönssonG, Skattum L and Truedsson L. 2014 Primary immunodeficiency in infectionprone children in southern Sweden: occurrence, clinical characteristics and immunological findings, BMC Immunology. 2014 ; 15:31. DOI : https://doi.org/10.1186/s12865-014-0031-6

11. The French national registry of primary immunodeficiency diseases. CEREDIH: The French PID study group. Clin Immunol. 2010 May; 135(2):264-72. DOI: 10.1016/j.clim.2010.02.021.

12. Gathmann B, Binder N, et coll. The European internet-based patient and research database for primary immunodeficiencies: update 2011 Clin Exp Immunol. 2012 July ; 169 (1) :70. DOI: 10.1111/j.1365-2249.2011.04542.x.

13. De Vries E, Driessen G. Educational paper: Primary immunodeficiencies in children: a diagnostic challenge. Eur J Pediatr. 2011;170(2):169-77. DOI: 10.1007/s00431-010-1358-5.

14. Bejaoui M, Barbouche MR, Sassi A, Larguche B, Miladi N, Bouguerra A, Dellagi K. 1997Primary immunodeficiency in Tunisia: study of 152 cases Arch Pediatr. 1997 ; 4(9) : 827-31. DOI:1016/s0929$693 x(97) 88145-6$

15. Reda SM, Afifi HM, Amine MM. 2009. Primary immunodeficiency diseases in Egyptian children: a single center study. Clin. Immunol. 2009. 29: 343- 351. DOI: 10.1007/s10875-008-9260-x.

16. Modell V, Knaus M, Modell F, Roifman C, Orange J, Notarangelo LD. Global overview of primary immunodeficiencies: a report from Jeffrey Modell Centers worldwide focused on diagnosis, treatment, and discovery. Immunol Res. 2014 Oct; 60 (1):132-44. DOI: 10.1007/s12026-014-8498-z.

17. Philippa Kirkpatrick \& Sean Riminton. Primary Immunodeficiency Diseases in Australia and New Zealand. J Clin Immunol 2007; 27:517 - 524. DOI:1007/s10875-007-9105-Z

18. Bittles $\mathrm{AH}$, Black ML. The impact of consanguinity on neonatal and infant health. Early Hum Dev. 2010 ; 86(11) : 737-41. DOI: 10.1016/j.earlhumdev.2010.08.003.

19. Hadizadeh H, Salehi M, Khoramnejad S, Vosoughi K, Rezaei The association between parental consanguinity and primary immunodeficiency diseases: A systematic review and meta- analysis. Pediatr Allergy Immunol. 2017 ; 28(3) : 280-287. DOI: 10.1111/pai.12685.

20. Barbouche MR, Galal N, Ben-Mustapha I, Jeddane L, Mellouli F, Ailal F, Bejaoui M, Boutros J, Marsaf A, Bousfiha A. A. Primary immunodeficiencies in highly consanguineous North African populations. Ann N Y Acad Sci. 2011 ; 1238 : 42-52. DOI: 10.1111/j.1749-6632.2011.06260.x.

21. Naidoo R, Ungerer L, Cooper M, Piennar S, Eley B.S. Primary Immunodeficiencies: a 27 year Review at a Tertiary Paediatric Hospital in Cape Town, South Africa. J Clin Immunol (2011) 31:99-105. DOI: https://doi.org/10.1007/s10875-010-9465-7

Page $11 / 13$ 
22. Al Herz W. Primary immunodeficiency Disorders in Kuwait: First Report from Kuwait National Primary immunodeficiency Registry (2004-2006) J Clin Immunol. 2007 ; 28 : 186-93. DOI: 10.1007/s10875007-9144-5.

\section{Figures}

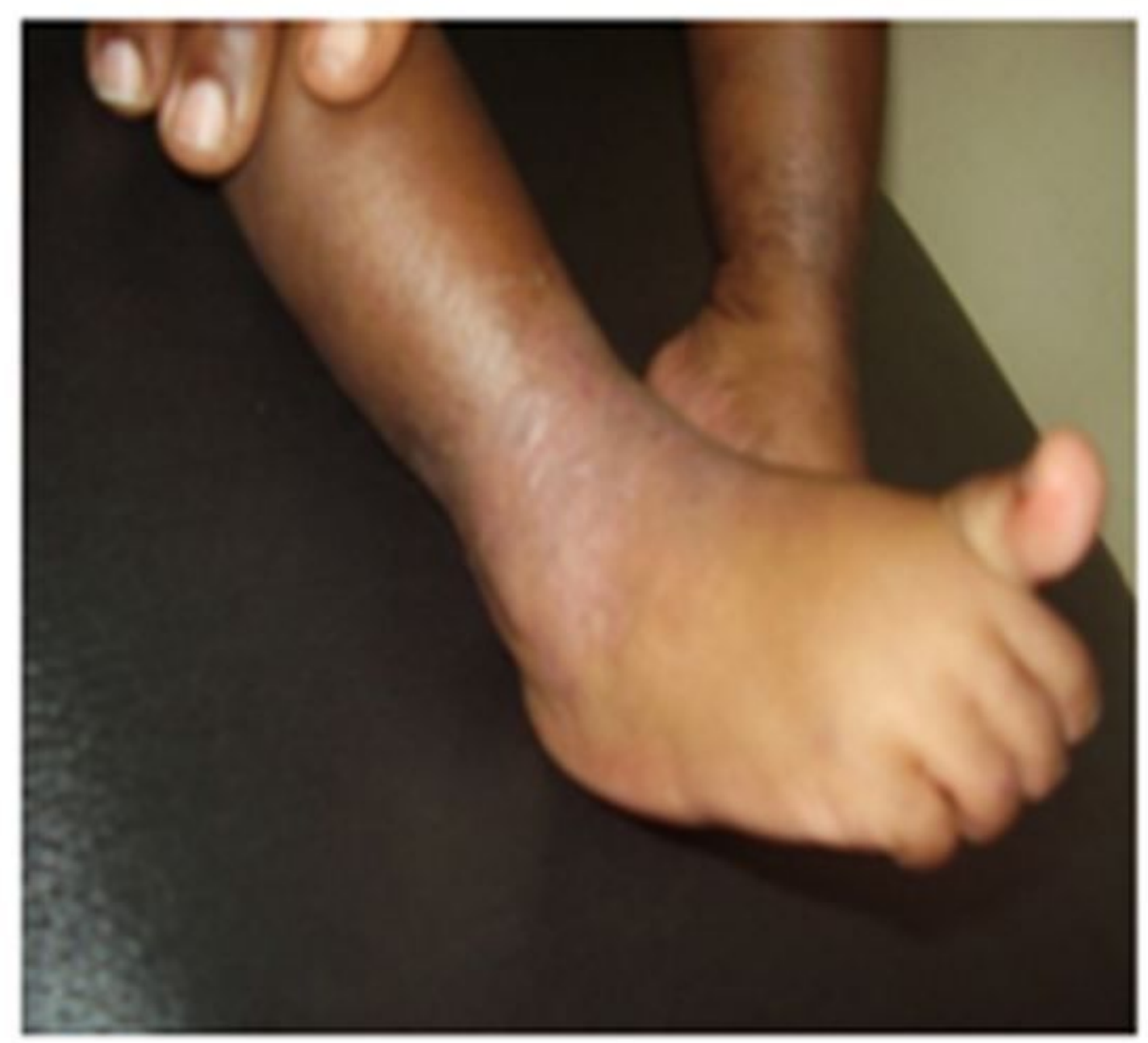

Figure 1

eczema in WAS patient 


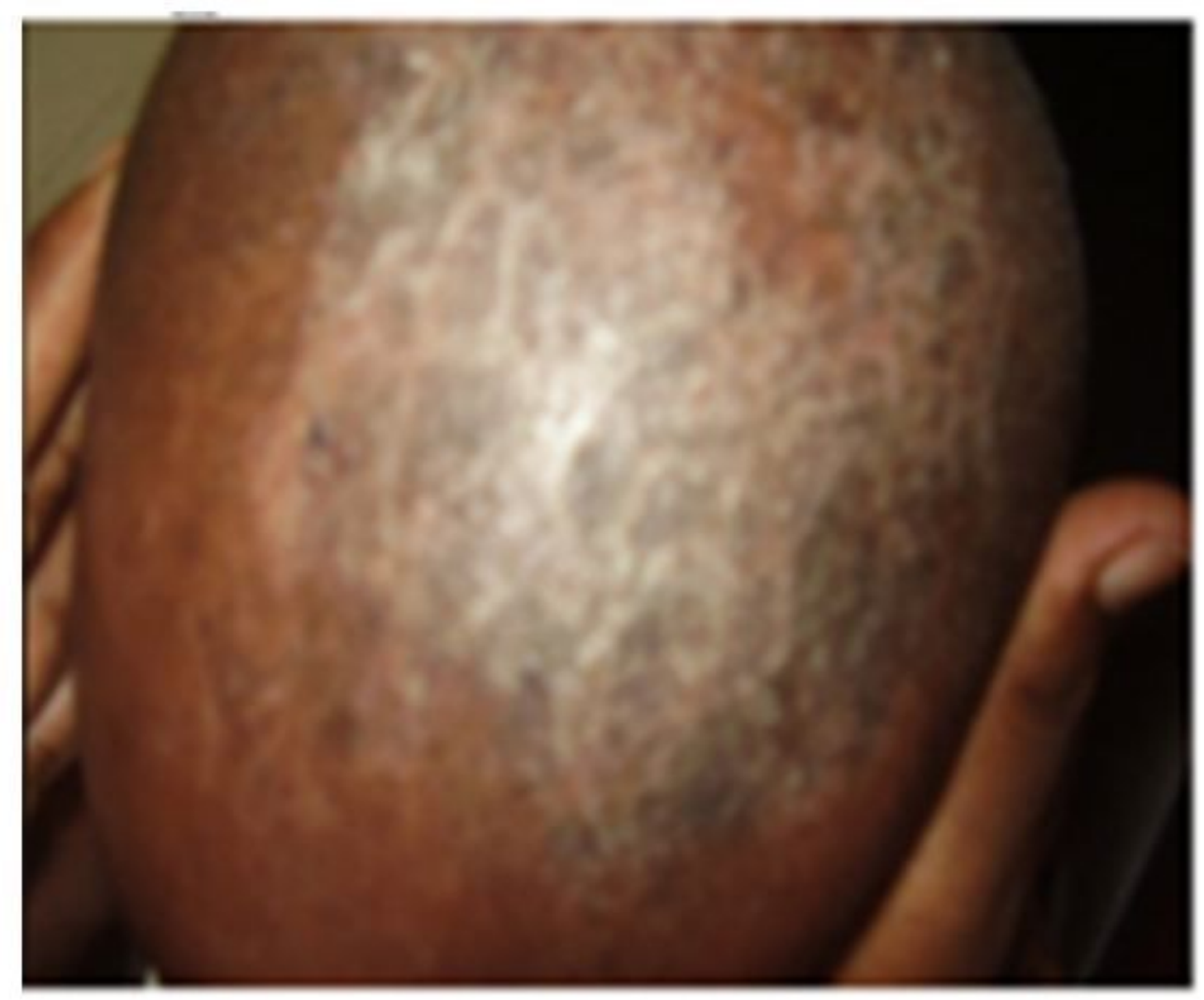

Figure 2

haemorrhagic scabs on the scalp of WAS

\section{Supplementary Files}

This is a list of supplementary files associated with this preprint. Click to download.

- additionalfiles.docx 